\title{
TOTAL GASTRECTOMY
}

\author{
By Terence KenNedy, M.S.(Lond.), F.R.C.S. \\ The Royal Victoria Hospital, Belfast
}

Though the stomach is a large and important organ, it is not absolutely essential to life, and a minority of patients after total gastrectomy enjoy remarkably good health for long periods. The majority of totally gastrectomized patients, however, suffer considerable disability, many becoming ' agastric cripples.' The operation is one, therefore, that should not be undertaken when there is a satisfactory alternative. The operative technique, with particular regard to the method of anastomosis, is of great importance and careful long-term post-operative management is essential.

The purpose of this paper is to examine some of the more important problems encountered.

\section{Indications for the Operation \\ I. Carcinoma}

The treatment of choice for carcinoma of the stomach is undoubtedly a radical partial gastrectomy. Usually this will involve removal of the distal part of the stomach. When the growth is limited to the cardia or fundus it will generally be treated by a radical proximal partial gastrectomy, though in such a case a total gastrectomy would be more radical, as it would allow dissection of the sub-pyloric and supraduodenal lymphatic glands.

Thus, total gastrectomy is essential only in those cases where the growth extends to within $I$ in. of the cardia and $2 \frac{1}{2}$ in. of the pylorus at the same time. The reason for the larger measurement at the distal end is that a pyloric remnant less than $I \frac{1}{2}$ in. in length, measured along the lesser curvature, is valueless, and section of the stomach must never be made less than $I$ in. from the edge of the tumour. At the proximal end, however, it is worth preserving even a small fringe of normal stomach, provided that this does not prejudice the chance of curing the disease.

When, at laparotomy, a carcinoma of stomach is found to be incurable on account of liver metastases, peritoneal spread, or irremovable glands, nearly all surgeons would agree that a palliative partial gastrectomy is very well worth while. Palliative total gastrectomy is, however, of doubtful value, in view of the higher immediate mortality and the considerable risk of severe nutritional disturbances.
It will be seen that total gastrectomy is par-ळ ticularly indicated for linitis plastica, extensive $\vec{O}$ ulcerating or infiltrating tumours of the body of $\overrightarrow{-}$ the stomach and multiple tumours, where there is ${ }_{\sigma}$ at least some prospect of cure.

\section{Sarcoma}

Sarcoma may be treated by total gastrectomy, but many cases, particularly of leiomyosarcoma, $\stackrel{+}{\circ}$ grow to a large size extra-gastrically, whilst in- $\overrightarrow{.}$ volving only a small area of stomach wall; $\operatorname{such}_{\infty}^{\omega}$ cases may be adequately treated by partialo gastrectomy.

\section{Gastric Polyposis}

Benign gastric polyps may be multiple and all $\stackrel{\widehat{\supset}}{\mathcal{1}}$ should be removed because of the risk of haemob $\overrightarrow{0}$ rhage and of carcinomatous change. When to whole, or nearly the whole, of the stomach involved total gastrectomy is indicated.

\section{Haematemesis from Multiple Gastric Erosions}

The management of these cases is always difficult $\stackrel{\circ}{\Phi}$ and, when operation is undertaken, most surgeons would prefer a subtotal gastrectomy, though some advocate total gastrectomy as the treatment of choice. If, in such a case, haemorrhage persists after a high partial gastrectomy, and no other cause can be found, then total gastrectomy must be seriously considered.

\section{Intractable Recurrent Peptic Ulceration}

Total gastrectomy for benign peptic ulcers is occasionally performed in error-a simple gastric 0 ulcer being thought to be carcinomatous, as in one of my cases. This error appears to be fairly common.

Deliberate total gastrectomy for recurrent peptic $\widetilde{N}$ ulcer behaving in a 'malignant' fashion has also N been advocated (Balint, J. A., and Gummer, N J. W. P.). Few surgeons would support their ${ }^{\omega}$ thesis that such drastic treatment is often, if ever, necessary for a peptic ulcer.

The names of Zollinger and Ellison are asso- $\stackrel{D}{\rightarrow}$ ciated with a syndrome in which recurrent peptic $\vec{\nabla}$ ulceration, with very high gastric acidity, is associated with a non-insulin-secreting tumour of $\stackrel{\mathbb{Q}}{\mathbb{Q}}$ 
pancreatic islet tissue. They recommend that the pancreatic tumour should be removed and total gastrectomy performed in addition.

If the pancreatic tumour is indeed the cause of the condition, and if it is removed, then it is difficult to see why any gastric mucosa should also be removed.

\section{Technique}

I. Approach

The operation can be done through three types of incision-a left thoracotomy, a left abdominothoracic incision, or a purely abdominal incision, either midline, left or right paramedian, or even a Kocher-type incision on the left side.

The dissection involved is extensive and the suturing must be meticulously careful, so that wide exposure is an essential prerequisite of success. There is no doubt that a left abdominothoracic incision, splitting the diaphragm, gives the most complete exposure, and is the route most generally favoured.

However, it has certain disadvantages. In old and frail patients, as many of these are, the exposure itself causes more shock than a purely abdominal incision. It is also more liable to cause troublesome or even dangerous pulmonary complications. Furthermore, though most thoracic surgeons would deny this, any incision with resection of a rib, or section of the costal margin, may cause severe and protracted wound pain. One of my patients became addicted to drugs as a result of this pain, which still persists after more than seven years.

Pure thoracotomy has the disadvantage of giving poor access to the duodenum, and a Kochers incision gives poor access to the abdominal oesophagus.

A vertical epigastric incision is generally entirely satisfactory, provided that the costal angle is not abnormally narrow, and that the patient is not unduly obese. Should the xiphisternum impede access it may readily be removed. If access is still inadequate, the lower part of the sternum may be split. Mobilization and dislocation of the left lobe of the liver will improve access to the oesophageal hiatus, but it is not usually necessary. It is simple to resect at least $\mathrm{I}$ in. of oesophagus and still make a satisfactory anastomosis below the diaphragm, but if the primary tumour extends above the cardia, then resection by a purely abdominal incision is likely to be inadequate, and the abdomino-thoracic route should be chosen.

\section{The Extent of the Resection}

Some surgeons leave a fringe of stomach at the upper end to facilitate anastomosis, yet describe their operation as a 'total gastrectomy.' Such cases should never be included in any discussio on total gastrectomy. The object of the operation when applied to cancer, is to remove the wholg stomach and as much of its lymphatic drainage asis reasonable, without unduly increasing the hazards. If those cases with liver metastases of widespread peritoneal involvement are excluded? it is involvement of the left gastric group of glands $\frac{\sigma}{5}$. near the upper border of the pancreas, which most often renders a growth incurable. If the body an $\$$ tail of the pancreas are resected with the stomacto and spleen, this group of glands is resected more radically. Partial pancreatectomy, however, adds. considerably to the operative mortality and i $\vec{\delta}$ probably not justifiable; if these glands are nos involved it is unnecessary, if they are involved the case is probably incurable.

In all cases both right and left gastric vessels and glands are resected as high as possible, the spleerif and the whole of the great omentum and gastrohepatic omentum are always removed. The rightw gastro-epiploic vessels and accompanying glandsీ are resected flush with the head of the pancreas? Removal of the upper peritoneal coat of the mesocolon and the peritoneum over the pancreaso in fact the posterior peritoneum of the lesser saco is often advocated and is theoretically desirable $\frac{1}{i 0}$ it is not always easy to resećt this completesy though it is readily done on the right side.

\section{Type of Anastomosis}

Perhaps the most important part of the operation is the choice of a suitable method of restoration of continuity, and its careful execution. Some year\& ago leakage from an anastomosis using the oeso $\overrightarrow{\vec{B}}$ phagus was frequent. Appreciation that the mucosa of the oesophagus is the strongest, and $\mathrm{d}_{\overrightarrow{7}}$ therefore, most vital, layer and the general adop? tion of interrupted non-absorbable suture ma을 terials, have largely abolished this risk. Fine. linen or silk may be used and my preference is fo the use of No. 90 linen in two layers of interrupted mattress sutures.

Second in importance to care in suturing tech nique comes the choice of the type of anastomosis used. The choice is wide, including oesophago duodenal anastomosis, either direct or with an. interposed loop of jejunum or transverse colon end-to-side anastomosis of oesophagus to a loop of jejunum, with or without an entero-anastomosis and end-to-end or end-to-side anastomosis of oesophagus to a Roux loop of jejunum.

The simplest method, because it involves onl one suture line, and artistically the most satisfyingo is the direct oesophago-duodenal anastomosis. Contrary to the opinion of many surgeons, this is in fact, an easy technique, possible in nearly ever case, unless a considerable length of oesophaguo क्ष 


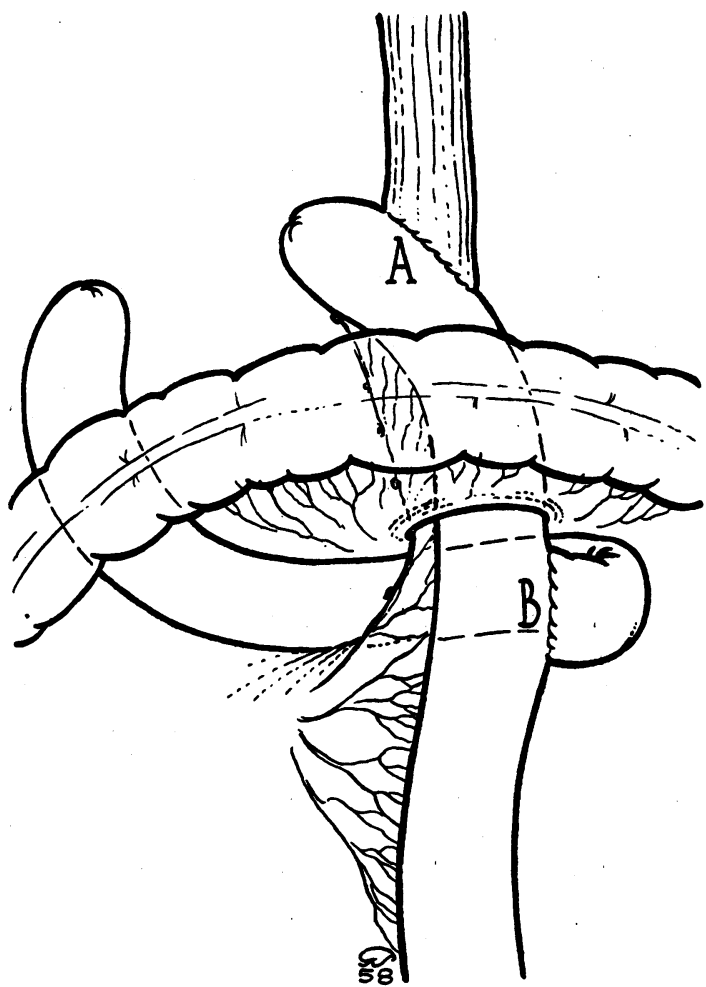

Figs. I and 2.-With a Roux loop, if the distance between $A$ and $B$ is short, the result is unsatisfactory due to oesophageal regurgitation. This can be prevented, without extra mobilization, by making the T-junction, $B$, a few inches lower, as in

Fig. 2. An end-to-end anastomosis at $\mathrm{A}$ is more satisfactory than an end-to-end-union.

has been resected. Tension on the anastomosis is relieved by mobilization of the duodenum and head of the pancreas after division of the peritoneum lateral to the second part of the duodenum. This mobilization, which is vital, allows the duodenum and the head of the pancreas to be swung upwards and to the left.

To bridge the gap between oesophagus and duodenum, a loop of jejunum or transverse colon has been interposed. This is not generally essential and greatly adds to the risk; three anastomoses are involved and the circulation of the isolated segment may be damaged, resulting in necrosis and leakage.

Second place for simplicity goes to the end-toside anastomosis of oesophagus to a jejunal loop, which may be brought up either in front of the colon or through the mesocolon. Generally an entero-anastomosis is added to diminish the risks of regurgitation.

The Roux loop is now probably the most popular method, and in my opinion, it is undoubtedly the best. Allison has given an excellent

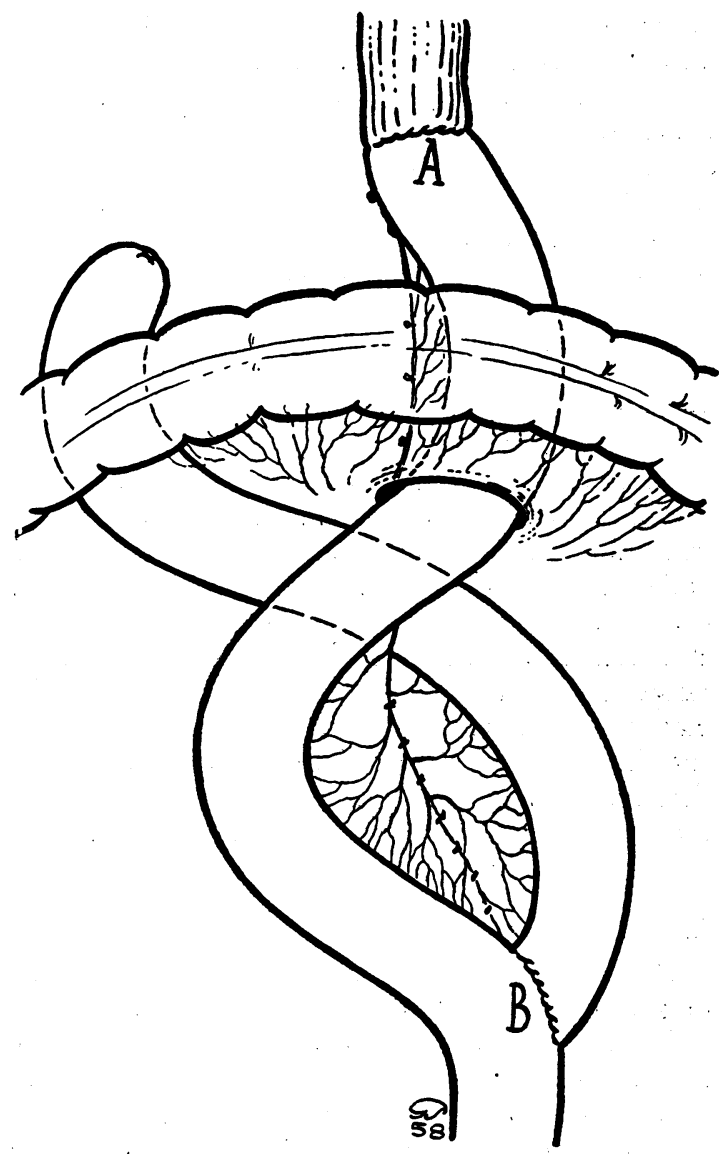

description of the method of constructing such a loop. It should be adequate in length, and is best brought up through the mesocolon. The quest for extra length in the upward or efferent limb may lead to excessive division of mesenteric vessels, causing gangrene and leakage at the site of anastomosis. This occurred in three of Allison's 6I cases where a Roux loop was anastomosed to oesophagus for malignant lesions. Extensive division of the mesentery is necessary to gain sufficient length for a high oesophago-jejunal anastomosis at, or above, the level of the aortic arch. In total gastrectomy, however, the jejunum can easily be brought up to the oesophagus without extra mobilization. It is important that the oesophagojejunal anastomosis should be at least ro in. above the end-to-side jejuno-jejunal anastomosis, to minimize the risk of regurgitation of bile and pancreatic juice into the oesophagus. This distance can easily be increased by inserting the proximal jejunal loop into the efferent loop at a lower level (Figs. I and 2). The proximal loop is always brought round behind the vertical loop and anas- 
tomosed to its left side; this allows the vertical limb to lie comfortably without distortion.

Sometimes the jejunal loop takes up an awkward curve, so that it is simpler to close its end and to anastomose the oesophagus to the side of the jejunal loop an inch or two proximal to its end. My own experience of this has been unsatisfactory. There has been much more oesophageal regurgitation, perhaps because the efferent limb of the loop is thereby shortened.

\section{Results \\ Immediate Mortality}

This will, of course, vary with the surgeon's criteria of operability and his indications for the operation. It will be adversely affected by such added measures as partial pancreatectomy. My own mortality has been just under ro per cent. Representative mortality rates in the literature range between 8 and $2 \mathrm{I}$ per cent. (Marshall; Ransom; Waugh et al.). It is of some interest that in the series with the best mortality the abdominal approach was used, whereas in that with the worst mortality the abdomino-thoracic approach was always used.

\section{Survival Rates}

It is not intended in this paper to discuss the long-term survival rates in detail. The factors influencing immediate mortality will obviously be important here-also. Marshall reports a five-year survival rate of 14 .I per cent. in a large series of cases of carcinoma. He used total gastrectomy in roughly one third of his cases, more than would most surgeons, so that his figures are probably better than average. Ransom had only four survivors in 59 cases. In my series there were only six total gastrectomies for carcinoma done more than five years ago and two remain alive at $7 \frac{1}{2}$ and $5 \frac{1}{2}$ years respectively.

\section{Oesophageal Regurgitation}

This is a frequent and troublesome complication. It is uncommon with a well-fashioned Roux loop, common with an end-to-side anastomosis using a simple jejunal loop, and almost invariable with direct oesophago-duodenal anastomosis.

My experience with the latter method is interesting. There were six cases with one operative death. One palliative resection had moderately severe oesophagitis, but died after one year. One young woman, $\mathrm{I} \frac{1}{2}$ years after resection for sarcoma, has severe oesophagitis and has had two small haematemeses. One man was crippled with oesophagitis and dysphagia for two years. His oesophago-duodenal anastomosis was then undone and a Roux loop was fashioned. He immediately improved dramatically, gaining over I st. in weight.
For a year he remained well till he developed reas currence and died $3 \frac{1}{2}$ years after resection. The fifth patient, a woman with a benign lesion mistaken for a carcinoma - the only one in my series-was crippled with oesophagitis and dys: phagia for three years, during which time she hap had recurrent episodes of bleeding and anaemis Oesophagoscopy showed ulceration and stricture above her anastomosis. This was resected and Roux loop anastomosis performed. The resecte $\$$ lower oesophagus contained two large 'tryptic ulcers. Improvement was dramatic and she gained $\mathrm{I} \frac{1}{2} \mathrm{st}$. in weight. She remains alive $2 \frac{1}{2}$ years later but still has mild oesophagitis, perhaps because att end-to-side anastomosis was used.

The sixth case was a man, aged sixty-nine, who had persistent mild symptoms of oesophagitis, with. mild inflammatory changes seen on repeated oesoo phagoscopy. After more than five years he des veloped severe dysphagia and was found to have recurrent tumour at the anastomosis. This has subsequently been resected, a surprisingly easf procedure, and he remains well with a Roux loops

Conversely, I have a number of patients with Roux loops who have survived varying periods us to $3 \frac{1}{2}$ years, who remain well without any oesoof phagitis or gastric symptoms of any kind.

\section{' Dumping'}

The dumping syndrome, as seen after partid gastrectomy, may occur after total gastrectomy but it is surprising, perhaps, that only a minorit of patients are affected. Advice to eat small bu frequent meals, and to drink fluids only between meals, is usually all that is needed.

\section{Deficiency States}

It is hardly surprising that these are ver. common. Iron deficiency anaemia is commong particularly in women, but is usually easily con? trolled. It is now recognized that every patien who survives long enough will eventually develog megaloblastic anaemia, as happened in all my earlo cases. Vitamin $B_{12}$, given prophylactically in doses of $100 \mu \mathrm{g}$. monthly will prevent this Perhaps the newer vitamin $B_{12}$ peptide comples preparations, which can be taken by mouth, wi prove adequate. In established cases larger doses will be required. Occasionally the addition of folic acid, iron and even ascorbic acid may be necessary (Welbourn, Nelson and Zacharias\%. These workers have stressed that the onset may be insidious, macrocytosis preceding the developmer of anaemia, so that a careful haematologica follow-up is essential. Haemoglobin estimations alone are unreliable, and blood films must be studied.

Other vitamin deficiencies are common, pa尽 
ticularly that of riboflavin. Ascorbic acid and vitamin A supplements may also be required. Hypoproteinaemia occasionally occurs in longterm survivors after total gastrectomy, and this may lead to starvation oedema. This has been a striking feature in one of my cases, developing after about two years. Vigorous treatment has produced improvement, but he still has some oedema after $7 \frac{1}{2}$ years.

\section{Weight Loss}

This is likely to be severe and intractable after total gastrectomy. Probably diminished food intake is of greater importance than poor absorption. Oesophagitis is a common and important cause of weight loss, which becomes extreme when the condition progresses sufficiently to cause dysphagia (Fig. 3). Generally the long-term survivors will be from $\mathrm{I}$ to 2 st. below their standard weight, though with Roux anastomoses they may remain only a few pounds below their normal weight.

This weight loss is not necessarily a serious matter, but malnutrition may lead to the development of pulmonary tuberculosis, as after partial gastrectomy. Routine annual chest X-ray, therefore, is highly desirable.

In treatment, various measures are available, of which the most valuable is advice and supervision by a skilled dietitian (Johnston, Welbourn and Acheson). The object is to increase the calorie intake, and in particular the protein intake, with supplements of milk, eggs, meat, etc. Financial help may be needed. Useful anabolic agents include methyltestosterone and iproniazid. Methyltestosterone in a dosage of $25 \mathrm{mg}$. daily may be used, but in women it must be used with caution, because of the risk of undesirable virilization.

\section{Conclusion}

No patient should be submitted to total gastrectomy without careful consideration of the alternatives.

An abdominal incision usually gives adequate exposure. The Roux loop makes the most satisfactory anastomosis. Because of the invariable oesophagitis, direct oesophago-duodenostomy is condemned.

$\mathrm{Re}$-operation is practicable and advisable when oesophagitis is troublesome.

Careful follow-up is advisable and all patients must take vitamin $\mathbf{B}_{\mathbf{1 2}}$ indefinitely. The help of a skilled dietitian is most rewarding.

\section{Acknowledgments}

My thanks are due to Mr. G. A. Smith for the drawings, to Mr. R. Woods for the photograph, and in particular to Professor R. B. Welbourn for

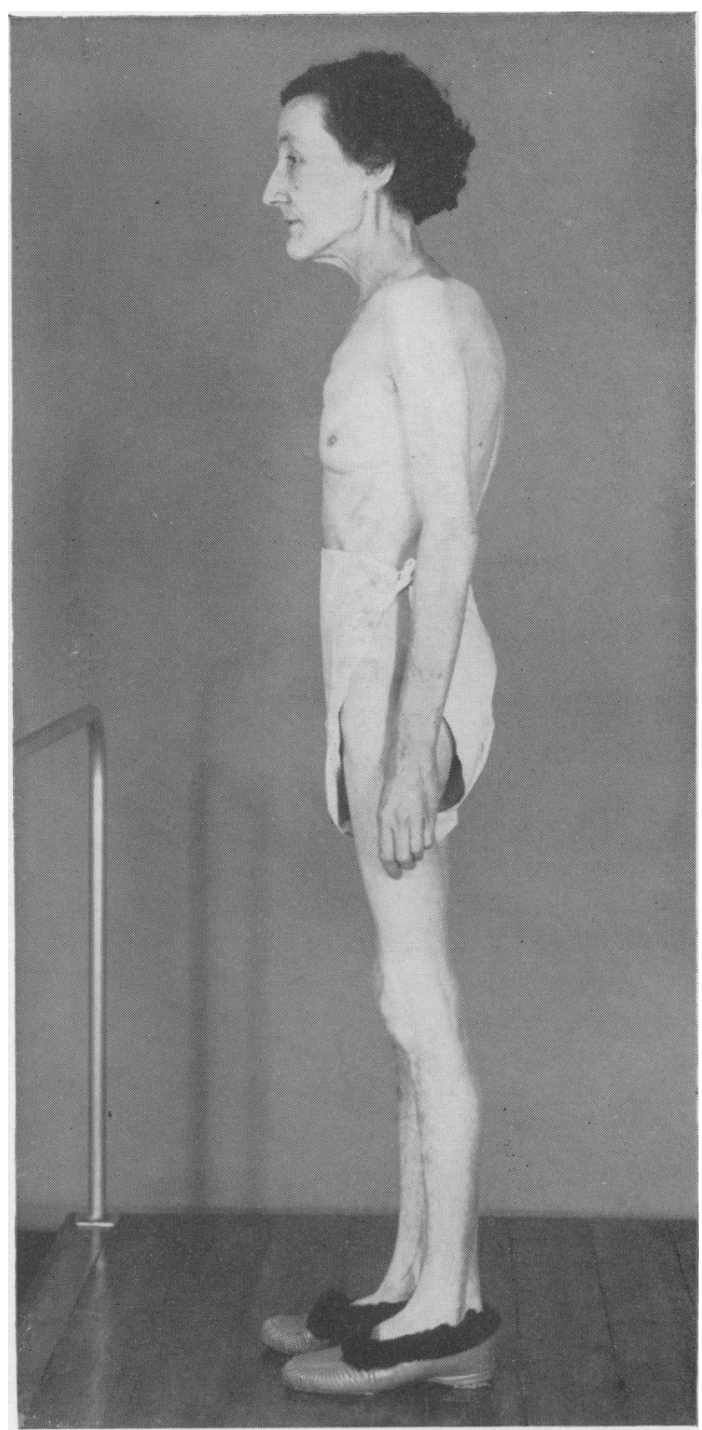

Fig. 3.-Severe emaciation three years after total gastrectomy, with oesophago-duodenostomy, for a benign lesion.

his help and advice in the post-operative management of many of my cases.

\section{BIBLIOGRAPHY}

ALlisON, P. R., and DA SILVA, L. T. (1953), Brit. F. Surg., 4I, 173 .

BALINT, J. A., and GUMMER, J. W. P. (1958), Lancet, i, 1044 JOHNSTON, I. D. A., WELBOURN, R., and ACHESON, K. (1958), Ibid., i, 1242.

MARSHALL, S. F. (1956), Surg. Clin. N. America, 36, 66r.

RANSOM, H. K. (1956), Gastroenterology, 30, 191.

WAUGH, J. M., WALTERS, W., PRIESTLEY, J. T., JUDD, E. S., and HALLENBECK, G. A. (1957), Staff' Meetings Mayo Clinic, 32, 599.

WELBOURN, R. B., NELSON, M. G., and ZACHARIAS, F. J. (1956), Brit. F. Surg., 43, 422.

ZOLLINGER, R. H., \& ELLISON, E. H. (1956), Surgery, 40, 147. 